\title{
Inner-shell charge transfer in asymmetric ion-atom collisions
}

\author{
J. F. Reading, A. L. Ford, G. L. Swafford, and A. Fitchard \\ Cyclotron Institute and Physics Department, Texas A\&M University, College Station, Texas 77843
}

(Received 10 February 1978)

\begin{abstract}
A method used previously for inner-shell ionization in asymmetric ion-atom collisions is extended to include charge transfer between the target inner shells and projectile $K$ shell. We work in the energy range $\eta^{1 / 2}=\hbar v / Z_{N} e^{2} \lesssim 1$ and use an independent-electron model (Hartree-Fock) for the target. We treat the interaction with the projectile as a time-dependent perturbation due to a bare charge moving on a straightline path. Our method, as for ionization, is very efficient in that with our target-centered expansion of the system wave function, all requisite matrix elements needed at a particular projectile energy are pretabulated and used at all impact parameters. A critical feature of our results is the recognition of the importance of target continuum states of energy approximately equal to the kinetic energy (in the target frame) of the electron on the projectile, and the development of a method to properly include such resonance states in our pseudostate calculation. We present selected numerical results to illustrate our method and to demonstrate the projectile energy and nuclear charge dependence of the cross sections. A general feature of the results presented is that the computed cross sections are of the order of 0.3-0.5 times the Brinkman-Kramers. estimate. Simultaneously, we also compute cross sections for electron stripping of the projectile, and find them to be dominated by transitions to the above-described resonant continuum state of the target.
\end{abstract}

\section{INTRODUCTION}

Two different methods have been generally used to compute charge transfer processes when a fast ion impinges on an atom: approximation schemes ${ }^{1}$ and numerical calculations ${ }^{2}$ in a Hilbert basis space. A valid criticism of both methods as previously applied is that they are unreliable. Approximations by definition omit certain features present in the real problem, and it is usually quite difficult to estimate accurately the error so caused in the final answer. Numerical calculations, on the other hand, while in principle exact, are slow and very expensive to perform. Because of this, convergence in the number of basis states is difficult to establish and is probably rarely achieved.

Recently a very fast numerical method has been used for inner-shell ionization by fast ions. ${ }^{3}$ This method is designed to be useful in an asymmetric situation where the effective charge $Z_{p}$ on one of the centers is much smaller than the effective charge $Z_{N}$ on the other center. In this case, the interaction can be treated as a small perturbation. The time-dependent wave function is efficiently described by an expansion in a basis set centered on $Z_{N}$. In this scheme the matrix elements can be stored as a function of $R$, the internuclear separation, resulting in an increase by two orders of magnitude in speed over conventional approaches that use two or more centers. ${ }^{2}$ Convergence in the wave-function expansion can therefore be demonstrably achieved.

It is the purpose of this paper to extend this method to charge transfer processes. Our approach is to solve the equation

$$
\frac{i \hbar \partial \Psi^{(+)}}{\partial t}=\left[-\frac{\hbar^{2}}{2 m_{e}} \nabla_{r}^{2}+U(r)+W(R)+V(\overrightarrow{\mathrm{r}}-\overrightarrow{\mathrm{R}}(t))\right] \Psi^{(+)}
$$

by a time-development operator approach, ${ }^{3}$ and then to evaluate the $T$ matrix for charge transfer, $\left(\phi_{C T}^{(-)},(U+W) \Psi^{(+)}\right)$. Here $U(\vec{r})$ is the single-electron Hartree-Fock potential for the target atom. The vector $\vec{r}$ locates the electron relative to the target nucleus, which is held fixed at the origin. The projectile describes a classical predetermined path $\overrightarrow{\mathrm{R}}(t)$. Throughout this paper we consider $\overrightarrow{\mathrm{R}}$ $\equiv(\vec{B}, \vec{Z})=(\vec{B}, \vec{v} t)$, where $B$ is the impact parameter and $v$, the velocity of the projectile, is assumed constant. The function $V((\vec{r}-\vec{R}(t))$ is the interaction between the active electron and the projectile nucleus, and $W(R)$ is a function of the projectile-target internuclear separation. For any choice of $W(R)$, the ionization and charge transfer cross sections are independent of $W(R)$ if the calculation is carried out exactly. An example of a $n u$ merically appropriate $W(R)$ is one that cancels the asymptotic $Z_{p} / R$ behavior of $V$, thus making $V+W$ go to zero asymptotically faster than $Z_{p} / R$. This shorter range of $V+W$ greatly aids in the numerical stability of the requisite integrals involving $V+W$, as will be discussed in detail in Sec. III. The function $\phi_{\mathrm{CT}}^{(-)}$is a solution of Eq. (1) with $U+W$ set to zero, and in our present calculation represents the electron bound in the $K$ shell of the moving projectile with potential $V$. (Here, and throughout, we refer to the collision partner with the larger effective charge $Z_{N}$ as the "target," and that with the smaller effective charge $Z_{p}$ as the "projectile.") 
An interesting feature of our investigation has been the realization that a straightforward calculation of the $T$ matrix for charge transfer in a Hilbert basis (either one- or two-centered) will converge very slowly. The reason for this will be described in detail in Sec. II. Basically the problem is that charge transfer takes place most easily when there is very good energy matching. Thus an important charge-transfer mechanism is the following. The projectile, charge $Z_{p}$, captures a $K$-shell electron by first exciting it to a continuum state with matching energy $\left(\sim \frac{1}{2} m_{e} v^{2}\right)$ and then resonantly charge-transferring it. (We will later demonstrate with numerical results the importance of correctly including this mechanism in a charge-transfer calculation.) The energy region in the continuum of the target for which the latter process can occur is very narrow. Its width can be estimated from the uncertainty principle as $\Delta E \sim \hbar / t=\hbar v Z_{p} / a_{0}$. In a Hilbert space, however, the continuum of target states is replaced by a finite number of discrete states. The energy gap typically present in these positive energy pseudostates is of the order of the gap between the bound states of the system, i.e., $\gamma Z_{N}^{2} e^{2} / a_{0}$. Here $\gamma$ is some factor less than unity that depends on the number of basis states being used. In the region of interest here, where $\eta^{1 / 2}=\hbar v / Z_{N} e^{2} \leqslant 1$ and $Z_{N}$ $\gg Z_{p}$, the energy gap between the positive energy pseudostates can be much larger than the energy region for resonant charge transfer. Thus most diagonalizations of the target Hamiltonian in a Hilbert basis will produce discrete pseudoenergies $\epsilon_{n}$, which miss the resonant energy region. The corresponding charge transfer so calculated will not include any resonance contribution. On the other hand, as the basis is varied, by scaling with a nonlinear parameter or by increasing the number of states, it may accidentally happen that a pseudostate does fall in the resonant energy region. However, now the resonance contribution is overestimated. This is because the positive-energy pseudostates each represent an energy region in the continuum (of width roughly equal to the separation between adjacent pseudostate energies), and in this "straightforward" calculation the whole of such a region is being treated as being at a single resonant energy.

Increasing the number of pseudostates in the basis is not a practical means of solving this difficulty. Instead, we use a new method that treats more accurately the discrete replacement of the continuum states.

This is described in Secs. II and III. In Sec. IV we apply it to calculate the charge-transfer contribution to $K(L)$-shell hole production by light ions incident on nonrelativistic atoms treated in the
Hartree-Fock approximation. Generally, we show that charge transfer between bound states has an approximate $Z_{p}^{5}$ variation with projectile charge, and a magnitude between 0.3 and 0.5 of the Brinkman-Kramers estimate. Comparison is made to experiment. Because of time-reversal symmetry, we also calculate simultaneously the cross section for removal ("stripping") of a $K$-shell electron from a light projectile by a heavier target. We present results for this process, and discuss its $Z_{p}$ behavior. We also note that such electron stripping from the small- $Z$ collision partner is dominated by resonant charge transfer into the continuum of the target.

\section{CONTINUUM STATES AND PSEUDOSTATES}

A basic relationship for formal manipulations in scattering perturbation theory is

$$
\begin{aligned}
& r^{-2} \delta\left(r-r^{\prime}\right)-\sum_{m} \chi_{m}^{*}(r) \chi_{m}\left(r^{\prime}\right) \\
& =\int_{0}^{\infty} \chi_{E}^{*}(r) \chi_{E}\left(r^{\prime}\right) \rho(E) d E .
\end{aligned}
$$

Here $\chi_{E}(r)$ and $\chi_{m}(r)$ are the true continuum and bound state wave functions, respectively, of the unperturbed Hamiltonian $H_{e}$, viz.,

$$
\begin{aligned}
H_{e} \chi_{E}(r) & =\left[-\frac{\hbar^{2}}{2 m_{e}}\left(\frac{1}{r^{2}} \frac{d}{d r} r^{2} \frac{d}{d r}+\frac{l(l+1)}{r^{2}}\right)+U(r)\right] \chi_{E}(r) \\
& =E \chi_{E}(r) .
\end{aligned}
$$

The wave functions are of definite angular momentum $l$, and $\rho(E)$ is the density of states. This closure relationship is replaced in a standard coupledstate calculation using pseudostates by an approximate one

$$
r^{-2} \delta\left(r-r^{\prime}\right)-\sum_{\substack{m \\ \epsilon_{m}<0}} \phi_{m}^{*}(r) \phi_{m}\left(r^{\prime}\right)=\sum_{\substack{n \\ \epsilon_{n}>0}} \phi_{n}^{*}(r) \phi_{\cdot n}\left(r^{\prime}\right)
$$

where here $\phi_{n}(r)$ are normalizable functions expanded in a truncated set of Hilbert basis states, which we represent by the projection operator $P$. Hence

$$
P H_{e} P \phi_{n}=\epsilon_{n} \phi_{n} .
$$

For a short-range potential $U$ we will have a finite number of bound states $\chi_{m}(r)$, and as the basis states are increased in number, these bound states will be accurately reproduced by the $\phi_{n}$ 's. The continuum states cannot be, but it is true that at the discrete positive energies $\epsilon_{n}$ that

$$
\chi_{\epsilon_{n}}(r) \approx N_{n} \phi_{n}(r)
$$

where $N_{n}$ is an $r$-independent factor. Of course, 
TABLE I. Demonstration of Eq. (10) for the relation between the continuum pseudoenergies and the widths $\Delta_{n} \cdot{ }^{\mathrm{a}}$

\begin{tabular}{|c|c|c|c|c|}
\hline & $\Delta_{n}$ & $\epsilon_{n}$ & $\frac{1}{2}\left(\Delta_{n}+\Delta_{n-1}\right)+\epsilon_{n-1}$ & Difference from $\epsilon_{n}(\%)$ \\
\hline \multirow[t]{5}{*}{7 -state basis } & 0.0 & 0.379 & & \\
\hline & 0.083 & 0.444 & 0.420 & 5.4 \\
\hline & 0.240 & 0.627 & 0.606 & 3.3 \\
\hline & 0.990 & 1.220 & 1.242 & 1.8 \\
\hline & 5.745 & 3.729 & 4.588 & 23 \\
\hline \multirow{7}{*}{9 -state basis } & 0.0 & 0.379 & & \\
\hline & 0.041 & 0.403 & 0.400 & 0.7 \\
\hline & 0.084 & 0.471 & 0.466 & 1.1 \\
\hline & 0.184 & 0.627 & 0.605 & 3.5 \\
\hline & 0.461 & 0.974 & 0.950 & 2.5 \\
\hline & 1.603 & 1.964 & 2.006 & 2.1 \\
\hline & 12.137 & 6.860 & 8.834 & 29 \\
\hline \multirow[t]{10}{*}{ 11-state basis } & 0.0 & 0.379 & & \\
\hline & 0.006 & 0.383 & 0.382 & 0.3 \\
\hline & 0.022 & 0.399 & 0.397 & 0.5 \\
\hline & 0.038 & 0.433 & 0.429 & 0.9 \\
\hline & 0.074 & 0.498 & 0.489 & 1.8 \\
\hline & 0.141 & 0.627 & 0.606 & 3.3 \\
\hline & 0.359 & 0.902 & 0.877 & 2.8 \\
\hline & 1.217 & 1.669 & 1.690 & 1.3 \\
\hline & 10.276 & 5.784 & 7.416 & 28 \\
\hline & $W_{n}$ & $X_{n}$ & $\frac{1}{2}\left(W_{n}+W_{n-1}\right)+X_{n-1}$ & Difference from $X_{n}(\%)$ \\
\hline \multirow{6}{*}{$\begin{array}{l}\text { 6-point } \\
\text { Laguerre } \\
\text { integration }^{b}\end{array}$} & 0.574 & 0.223 & & \\
\hline & 1.369 & 1.189 & 1.195 & 0.5 \\
\hline & 2.261 & 2.993 & 3.004 & 0.4 \\
\hline & 3.351 & 5.775 & 5.799 & 0.4 \\
\hline & 4.887 & 9.837 & 9.894 & 0.6 \\
\hline & 7.849 & 15.983 & 16.205 & 1.4 \\
\hline
\end{tabular}

${ }^{a}$ For titanium Hartree-Fock $s$ states. Energies are measured such that the $K$-shell orbital has zero energy. The energy $\epsilon=I_{K}=0.379$ has been added to the table in each case. All energies are in units of $Z_{N}^{2} e^{2} / a_{0}$.

${ }^{\mathrm{b}} \int_{0}^{\infty} g(x) d x=\sum_{\mathrm{r} i} W_{i} g\left(\boldsymbol{X}_{i}\right)$.

the identity of these two functions is only good in the region restricted to not-too-large $r$ as $\phi_{n}(r)$ is bounded whereas $\chi_{\epsilon_{n}}(r)$ satisfies scattering boundary conditions. ${ }^{n}$ In two recent papers, ${ }^{4}$ for example, it has been shown that it is possible to accurately reproduce $\chi_{\epsilon_{n}}(r)$ with a $\phi_{n}(r)$ over a satisfactorily large range of $r$. It is also straightforward to find $N_{n}$ by noting that

$\chi_{\epsilon_{n}}(r)=j_{l}\left(k_{n} r\right)+\int_{0}^{\infty} G_{\epsilon_{n}}\left(r, r^{\prime}\right) U\left(r^{\prime}\right) \chi_{\epsilon_{n}}\left(r^{\prime}\right) r^{\prime 2} d r^{\prime}$

and substituting from Eq. (6) for $\chi_{n}(r)$. We can then find $N_{n}$ by various methods. ${ }^{4}$

To understand more thoroughly the replacement of the integral in Eq. (2) by the sum of Eq. (4), we postulate that the scheme is like any other integration rule in that certain abscissas $\epsilon_{n}$ are weighted with widths $\Delta_{n}$ and a finite sum produced which approximates the integral. That is,

$$
\begin{aligned}
\int_{0}^{\infty} \chi_{E}^{*}(r) \chi_{E}\left(r^{\prime}\right) \rho(E) & d E \\
& =\sum_{n} \int_{\epsilon_{n}-\Delta_{n} / 2}^{{ }^{\epsilon} n^{+\Delta_{n}} / 2} \chi_{E}^{*}(r) \chi_{E}\left(r^{\prime}\right) \rho(E) d E \\
& \approx \sum_{n} \chi_{\epsilon_{n}}^{*}(r) \chi_{\epsilon_{n}}\left(r^{\prime}\right) \rho\left(\epsilon_{n}\right) \Delta_{n} \\
& \approx \sum_{n} N_{n}^{2} \Delta_{n} \rho\left(\epsilon_{n}\right) \phi_{n}^{*}(r) \phi_{n}\left(r^{\prime}\right)
\end{aligned}
$$

But if Eq. (4) is to hold, then this says that 


$$
N_{n}^{2} \Delta_{n} \rho\left(\epsilon_{n}\right)=1 \text {. }
$$

As we know $N_{n}$, we can calculate $\Delta_{n}$ and find the weight $\Delta_{n}$ which the diagonalization of $P H_{e} P$ in our basis has provided for each pseudoenergy. ${ }^{5}$

A useful test of this postulate can be obtained by noting that if it is true then the whole energy range of $E$ must be spanned and there should be no gaps. That is, the relation

$$
\epsilon_{n} \approx \frac{1}{2}\left(\Delta_{n}+\Delta_{n-1}\right)+\epsilon_{n-1}
$$

should hold. In Table I we give an example of this rule for diagonalization of a Hartree-Fock Hamiltonian for $s$ states, in bases of 7, 9, and 11 states. The atom considered here is titanium. It is typical of the cases we consider in that it may be accurately treated nonrelativistically. In general we found that Eq. (10) is more closely obeyed as the size of the basis is increased. For comparison we examine the analogous rule for Laguerre integration. The $\epsilon_{n}$ and $\Delta_{n}$, as well as the $W_{i}$ and $X_{i}$ in Laguerre integration, can be observed to obey numerous other approximate relationships, for example that $\Delta_{n} \approx \frac{1}{2}\left(\epsilon_{n+1}-\epsilon_{n-1}\right)$.

Of course, this agreement is only an indication that our derivation is correct. It in no way constitutes a mathematical proof, which is far beyond the intent of this work. It has to fail, of course, for the last interval, which is infinite in extent. This in itself indicates the necessity noted in our previous work of making sure the basis chosen adequately spans the energy range of interest, for of course no finite rule can adequately replace every integral.

This brings us to the real point though. In a practical application we will always be interested in an energy weighted integration. For example, in the interaction picture for a time-dependent problem we are interested in exponentiating the Hamiltonian $H_{e}$. To do this we use closure and write

$$
\begin{aligned}
\exp \left(\frac{i H_{e} t}{\hbar}\right) r^{-2} \delta\left(r-r^{\prime}\right) \\
\equiv \int_{0}^{\infty} \exp \left(\frac{i E t}{\hbar}\right) \chi_{E}^{*}(r) \chi_{E}\left(r^{\prime}\right) \rho(E) d E \\
+\sum_{m} \exp \left(\frac{i E_{m} t}{\hbar}\right) \chi_{m}^{*}(r) \chi_{m}\left(r^{\prime}\right) \\
=\sum_{n, \text { all stat es }} \exp \left(\frac{i \epsilon_{n} t}{\hbar}\right) \phi_{n}^{*}(r) \phi_{n}\left(r^{\prime}\right)
\end{aligned}
$$

If our basis is adequate [a good test of which is that there are no gaps, i.e., that Eq. (10) is satisfied], then we may correctly replace $\chi_{\epsilon_{n}}$ by $N_{n} \phi_{n}(r)$ as discussed above. However, it is true that while our basis may have passed this rudi- mentary test, the scheme of Eq. (11) may still fail. For large enough “ $t$ ", i.e., when $\Delta_{n} t / \hbar z 1$, the oscillations caused by the exponentiation of $E$ may be too severe for Eq. (11) to work. Recognizing this, an alternative representation of $\exp \left(i H_{e} t / \hbar\right)$ is given by

$$
\begin{aligned}
r^{-2} \delta\left(r-r^{\prime}\right) & \exp \left(i H_{e} t / \hbar\right) \\
& \approx \sum_{n} \int_{\epsilon_{n}-\Delta_{n} / 2}^{\epsilon_{n^{+} \Delta_{n} / 2}} \exp (i E t / \hbar) N_{n}^{2} \phi_{n}^{*}(r) \phi_{n}\left(r^{\prime}\right) \\
& \times \rho\left(\epsilon_{n}\right) d E \\
& =\sum_{n} \exp \left(i \epsilon_{n} t \hbar\right) \phi_{n}^{*}(r) \phi_{n}\left(r^{\prime}\right) F_{n}
\end{aligned}
$$

where

$$
F_{n}=\frac{\sin \left(\Delta_{n} t / 2 \hbar\right)}{\Delta_{n} t / 2 \hbar} \equiv \frac{\sin \kappa}{\kappa} .
$$

Here we include the bound states in the sum, but, recognizing that as these are genuine discrete states, their $\Delta$ 's are zero.

Note that for small enough times $t$ the correction factors $F_{n}$ to be applied to the normal scheme of Eq. (11) are unity. For example, if we are interested in $K$-shell ionization, the order of magnitude of $t$ is given by $a_{0} /\left(Z_{N} v\right)$. Thus for this case, since $\Delta_{n} \approx \gamma Z_{N}^{2} e^{2} / a_{0}$ as pointed out in the introduction,

$$
\kappa=\gamma Z_{N} e^{2} / 2 \hbar v=\gamma / 2 \eta^{1 / 2} .
$$

The error in replacing the $F_{n}$ 's by unity is thus of the order of $\gamma^{2} / 24 \eta$. In the typical bases shown in Table I, $\gamma$ is of the order of 0.5 or less and a small error is made by this approximation. These then are the underpinning numerics on which the conventional coupled-state approach to the continuum problem rests.

For charge transfer in an asymmetric situation, the conventional procedure fails because large times are involved. The $K$-shell projectile bound state extends a distance $a_{0} / Z_{p}$, and the error now made in the conventional approach should be estimated from a time $a_{0} / Z_{p} v$. Thus the error in replacing $F_{n}$ by unity is a factor of $\left(Z_{N} / Z_{p}\right)^{2}$ larger. In Sec. III we use these ideas for a successfully convergent treatment of charge transfer.

\section{CHARGE TRANSFER}

Eventually we would hope to treat many-electron atom-atom collisions, but for now we restrict ourselves to the situation in which we have one electron and two nuclei. Thus the effective charges $Z_{p}$ and $Z_{N}$ we take to be the nuclear charges of the projectile and target. One of these, the projectile, we treat in a hydrogenic model, i.e., it is taken to be a proton or other bare ion. We also 
restrict ourselves in this paper to consideration of transitions to or from the $K$ shell of the projectile. The target nucleus is treated as a fully dressed neutral atom, and we calculate charge transfer to and from the Hartree-Fock $K$, $L$, etc., shells and the Hartree-Fock continuum of the target. Time-reversal symmetry implies that our calculations are as appropriate to electron stripping from a hydrogen atom by collision with a copper ion as they are to electron pickup by a proton from a copper atom.

The reason for the above choice of projectile and target potentials is that this model is entirely appropriate for calculating the contribution to $K$ shell hole production by bare ions incident on an atom. In an earlier paper ${ }^{6}$ in this work it was shown that as long as the atom under consideration could be treated in the independent particle model, then the cross section for $K$-shell hole production was given by paying no regard to the presence of the other electrons but rather by just considering excitation of a single $K$-shell electron above the
Fermi sea. In a recent paper ${ }^{7}$ it has been shown that this single-electron model also applies in the charge transfer contribution to $K$-shell hole production as long as the projectile states are accurately treated in the independent-particle model. The same statement can be made only approximately about calculations of cross sections for charge transfer accompanied by the production of $K$-shell holes. In these processes antisymmetry destroys the independence of the single electron transitions involved. We do not attempt to calculate detailed cross sections for such events in this preliminary work, and conclusions drawn as to the relevance of our results to such experiments ${ }^{8}$ should be treated with caution. Here then our calculations are only strictly appropriate to $K$-shell hole production or to genuine single-electron situations.

The probability amplitude for charge transfer from a target state of energy $E$ (measured from the $K$-shell energy) to the $K$-shell of the projectile incident at impact parameter $B$ is given by

$$
t_{\mathrm{CT}}^{E}=\eta^{-1 / 2}\left(\frac{2}{\sqrt{4 \pi}} f^{3 / 2}\right) \int_{-\infty}^{+\infty} \exp \left(i \lambda_{1} Z\right)\left(\int \exp (-f|\overrightarrow{\mathrm{R}}-\overrightarrow{\mathrm{r}}|) \exp \left(i \vec{\lambda}_{2} \cdot \overrightarrow{\mathrm{r}}\right)(\bar{U}+f \bar{W}) \Psi_{E}^{(+)}(\overrightarrow{\mathrm{r}}, \overrightarrow{\mathrm{R}}) d^{3} r\right) d Z \equiv t_{U}^{E}+t_{W}^{E}
$$

The cross section for such a process is obtained by integrating over impact parameter

$$
\sigma_{\mathrm{CT}}^{E}=2 \pi a_{N}^{2} \int_{0}^{\infty}\left|t_{\mathrm{CT}}^{E}\right|^{2} B d B
$$

In accord with the above comments an additional factor of $\mathbf{2}$ would be needed to account for the presence of two electrons in the initial state, for example, the $K$ shell, of the target. In Eq. (14), $R$ and $r$ are measured in units of $a_{N}=a_{0} / Z_{N}$. In this system of units

$$
f=Z_{\mathrm{p}} / Z_{N}, \quad \lambda_{2}=-\hbar v / Z_{N} e^{2} \equiv-\eta^{1 / 2},
$$

and

$$
\lambda_{1}=\frac{1}{2} \eta^{1 / 2}+\left(I_{K}-\frac{1}{2} f^{2}\right) / \eta^{1 / 2},
$$

where $I_{K}$ is the $K$-shell ionization potential for the target atom. The potentials are replaced by dimensionless quantities. Thus

$$
U \Rightarrow \bar{U}=-\frac{1}{r}+U_{\mathrm{HF}},
$$

where $-1 / r$ is the electron-nuclear attraction and
$U_{\mathrm{HF}}$ consists of the screening and exchange interactions of each electron with the others, treated in the Hartree-Fock approximation, and

$$
\begin{aligned}
& W \Rightarrow f \bar{W}=f[1-\exp (-R)] / R, \\
& V \Rightarrow f \bar{V}=-f /|\overrightarrow{\mathrm{R}}-\overrightarrow{\mathbf{r}}| .
\end{aligned}
$$

These potentials will be discussed in more detail shortly. We have pulled a factor $f$ out of $V$ and $W$ to facilitate later discussion of the $f$ dependence of the cross section. The wave function $\Psi^{(+)}$satisfies the equation

$$
\begin{aligned}
\Psi^{(+)}(\overrightarrow{\mathrm{r}}, Z)= & \chi_{E}(\overrightarrow{\mathrm{r}}) \exp \left(-i \lambda_{E} Z\right)-i f \exp \left(-i \bar{H}_{e} Z\right) \eta^{-1 / 2} \\
& \times \int_{-\infty}^{Z} \exp \left(i \bar{H}_{e} Z^{\prime}\right)[\bar{V}+\bar{W}] \\
& \times \Psi^{(+)}\left(\overrightarrow{\mathrm{r}}, Z^{\prime}\right) d Z^{\prime}
\end{aligned}
$$

and

$$
\vec{H}_{e} \chi_{E}=\left(a_{N} / \hbar v\right) H_{e} \chi_{E}=\left(a_{N} E / \hbar v\right) \chi_{E}=\lambda_{E} \chi_{E}
$$

defines $\bar{H}_{e}, \chi_{E}$, and $\lambda_{E}$. The first Born approximation for $\Psi_{E}^{(+)}$corresponds to taking the first term 
on the right-hand side of Eq. (16). The probability amplitudes computed with this first Born $\Psi^{(+)}$we will refer to as $t_{U, B}^{E}, t_{\mathrm{W}, B}^{E}$, and $t_{\mathrm{CT}, B}^{E}=t_{U, B}^{E}+t_{\mathrm{W}, B}^{E}$.

The function $\bar{W}(R)$ can be chosen for convenience, as the probability $\left|t_{\mathrm{CT}}^{E}\right|^{2}$ is independent of $W$ in an exact calculation, within a time-dependent approximation to the scattering such as ours. We tested our numerical procedures by demonstrating this to be the case. The choice of $\bar{W}(R)$ used for the results reported here was $\left(1-e^{-R}\right) / R .^{9}$ This has the property of keeping $\bar{V}+\bar{W}$ of $O\left(R^{-2}\right)$ as $R$ goes to infinity, a very useful result as we shall see later. This $\bar{W}(R)$ is also finite at the origin. A variety of other choices of $\bar{W}(R)$ have been used in first Born calculations of charge transfer. The well-known BK approximation of Brinkman and Kramers ${ }^{1}$ uses no $\bar{W}(R)$. Halpern and $\mathrm{Law}^{10}$ took $\bar{W}(R)$ to be the full projectile nucleus-target nucleus interaction, making $\bar{W}(R)=Z_{N} / R$ in the present units. [They referred to this choice of $W(R)$ as giving the "Jackson and Schiff amplitude," as an analogous full internuclear interaction was used by the latter authors in a treatment of $p+H$ charge transfer. ${ }^{11}$ ] Note that with either of these $\bar{W}(R)$, $\bar{V}+\bar{W}$ has an asymptotic $1 / R\left[\operatorname{or}\left(Z_{N}-1\right) / R\right]$ behavior. Thus with our methods either of these choices would be numerically inconvenient if the calculation is carried past first Born. A choice of $\bar{W}(R)$ somewhat similar to ours, $\bar{W}(R)=1 / R$, has been used by Omidvar et al., and yet another choice has been made by Band. ${ }^{12}$ The aim of all these choices is to minimize the higher Born terms, an ambition which has particular merit if one intends to neglect them. Our numerical and analytical investigations lead us to the conclusion that in this regard our choice of $\bar{W}(R)$ is superior to the others mentioned above. For all results reported here, our $\bar{W}(R)$ makes the first Born cross section $\sigma_{\mathrm{CT}, B}^{E}$ fairly close in magnitude (specific numerical examples will be given in Sec. IV) to the cross section we calculate with our accurate $\Psi_{E}^{(+)}$. As an example of the independence in our calculations of $\left|t_{\mathrm{CT}}^{E}\right|^{2}$ and $\sigma_{\mathrm{CT}}^{E}$ from the choice of $\bar{W}(R)$, consider $6-\mathrm{MeV} \alpha$-particle projectiles incident on titanium. (This target atom, projectile, and projectile energy choice is representative of those for which cross sections are to be presented in Sec. IV.) In addition to our usual $\bar{W}(R)=\left(1-e^{-R}\right) / R$ we also used

$$
\bar{W}(R)=\left\langle\phi_{1 s}(r)\left|\frac{1}{|\overrightarrow{\mathrm{R}}-\overrightarrow{\mathrm{r}}|}\right| \phi_{1 s}(r)\right\rangle,
$$

where $\phi_{1 s}$ is the Hartree-Fock $K$-shell orbital for titanium. This $W(R)$ is approximately $\left(1-e^{-2 R}\right) /$ $R-e^{-2 R}$ and makes $\left\langle\phi_{1 s}|\bar{V}+\bar{W}| \phi_{1 s}\right\rangle$ equal to zero. For this latter $\bar{W}(R)$, as compared to the former, $\sigma_{\mathrm{CT}, \mathrm{B}}^{E}$ for the target $K$-shell changed by about $25 \%$ whereas $\sigma_{\mathrm{CT}}^{E}$ changed by less than $1 \%$.

The target atom potential $\bar{U}(r)$ was written as the sum of two terms

$$
\bar{U}=-1 / r+U_{\mathrm{HF}},
$$

where $U_{\mathrm{HF}}$ contains the effects of screening and exchange due to the other electrons in the atom. This $U_{\mathrm{HF}}$ was represented as the Hartree-Fock potential $V_{\mathrm{HF}}$ from the compilation of Clementi and Roetti, ${ }^{13}$ expressed in the present units and expanded in the same set of basis functions used for expanding $\Psi_{E}^{(+)}$. That is,

$$
U_{\mathrm{HF}} \phi_{m}=\sum_{n}\left\langle\phi_{n}\left|V_{\mathrm{HF}}\right| \phi_{m}\right\rangle \phi_{n}
$$

The $-1 / r$ part of $U$ was treated separately so that the remainder would be finite at the origin and thus more easily expandable in our basis.

It is instructive to examine $t_{\mathrm{CT}}^{E}$ in the limit $Z_{p}$, or $f, \rightarrow 0$ with $Z_{N}$ held fixed. For direct collisions one is used to the idea that in the limit of small perturbation $\Psi_{E}^{(+)}(\overrightarrow{\mathrm{r}}, Z)-\Psi_{E}^{(+)}(\overrightarrow{\mathrm{r}},-\infty)$, i.e., the Born approximation should be accurate both for the wave function and amplitude. Consider, however, the result of replacing $\Psi_{E}^{(+)}(\overrightarrow{\mathrm{r}}, Z)$

by $\exp \left(-i \lambda_{E} Z\right) \chi_{E}(\overrightarrow{\mathrm{r}})$ in Eq. (14). In the $f \rightarrow 0$ limit, if one sets $f \bar{W}$ to zero and replaces $\exp (-f|\overrightarrow{\mathbf{R}}-\overrightarrow{\mathbf{r}}|)$ by unity, then the $Z$ integration is trivial and gives $t_{\mathrm{CT}}^{E} \propto \delta\left(\lambda_{1}-\lambda_{E}\right)$. If $\lambda_{1} \neq \lambda_{E}$, i.e., we are not in the continuum resonance region, then this lowest-order (in $f$ ) contribution to $t_{\mathrm{CT}}^{E}$ is zero. The first nonvanishing term in the first Born amplitude must come then from $f \bar{W}$ and from the term proportional to $f$ in the expansion of $\exp (-f|\overrightarrow{\mathbf{R}}-\overrightarrow{\mathbf{r}}|)$. In either case this produces an extra $f$, making in the asymmetric limit the first Born term the same order in $f$ as the second Born term. The importance of the second Born term in charge transfer has also been demonstrated by Drisko, ${ }^{14}$ who showed that in the limit of high energy the second Born term dominates over the first.

The above discussion also shows that at resonance, where $\lambda_{E}=\lambda_{1}$, the Born term $t_{U, B}^{E}$ will dominate. The physical meaning of this result is easy enough to understand in the time-reversed situation where the light projectile with a bound electron is incident on a heavy target. As the electron enters the strong target potential it loses contact with the projectile and enters a continuum state of the target with energy $\sim \frac{1}{2} m_{e} v^{2}+I_{K}$. (Note that if $E$, relative to the $K$ shell, is $\frac{1}{2} m_{e} v^{2}+I_{K}$, then $\lambda_{E}=\frac{1}{2} \eta^{1 / 2}+I_{K} / \eta^{1 / 2} \approx \lambda_{1}$.) As noted in Sec. I, the internal velocity of the electron in the projectile gives a natural width to this state. This width is now readily calculated by examining the $Z$ integration in Eq. (14) (with $f$ very small, but not 
zero). This gives an integral of the form (assuming $r \ll R$ in the region of interest)

$$
\begin{aligned}
\int_{-\infty}^{\infty} \exp (-f R) \exp \left[i\left(\lambda_{1}-\lambda_{E}\right) Z\right] d Z \\
=\frac{2 f B K_{1}\left\{B\left[f^{2}+\left(\lambda_{1}-\lambda_{E}\right)^{2}\right]^{1 / 2}\right\}}{\left[f^{2}+\left(\lambda_{1}-\lambda_{E}\right)^{2}\right]^{1 / 2}} \rightarrow \frac{2 f}{f^{2}+\left(\lambda_{1}-\lambda_{E}\right)^{2}} .
\end{aligned}
$$

The last expression is obtained in the limit that $f B$ and $\left(\lambda_{1}-\lambda_{E}\right) B$ are small. This factor is of great importance in the following discussion. In terms of energy it corresponds to a resonance of width $\Gamma$ where

$$
\Gamma=\hbar v f / a_{N}=Z_{p} \hbar v / a_{0}
$$

This is exactly what we estimated from the uncertainty principle in Sec. I. Note also the difference in the $B$ dependence of $t_{\mathrm{CT}}^{E}$ on and off resonance. For large $B, K_{1}(a B)$ behaves approximately like $e^{-a B}$ and thus causes $t_{\mathrm{CT}}^{E}$ to fall off in $B$ as $\exp \left[-\left(\lambda_{1}-\lambda_{E}\right) B\right]$ off resonance and as $\exp (-f B)$ on resonance. Thus the resonant $t_{\mathrm{CT}}^{E}$ "sticks out", much further in $B$. This gives an additional reason why $t_{U, B}^{E}$ dominates in $\sigma_{\mathrm{CT}}^{E}$ at resonance. At the larger $B, \bar{V}+\bar{W}$ is small and the first Born approximation for $\Psi_{E}^{(+)}$is accurate, and for large
$B, \bar{W}$ is much less than $\bar{U}$. These above-described qualitative features will be further discussed in terms of actual numerical results in Sec. IV.

As discussed in Sec. I, these continuum resonance states provide a natural set of window states for all other capture processes. However, the amplitude for resonance transfer [Eq. (17)], is a rapidly varying function of energy for small $f$ and thus cannot be accurately integrated with a practical number of pseudostate energies. Hence for the $\exp \left(-i \bar{H}_{e} Z\right)$ factor in Eq. (16) we must use the modified pseudostate expansion of Eq. (12).

The above comments are all based on a consideration of $t_{U}^{E}$. As $\bar{W}$ falls off as $1 / R$, large values of $Z$ are not nearly so important in $t_{W}^{E}$, and $t_{W}^{E}$ is a more slowly varying function of $\lambda_{E}$. Also note that whereas we have argued for a special treatment of the $\exp \left(-i \bar{H}_{e} Z\right)$ factor in Eq. (16), there is a $\exp \left(i \bar{H}_{e} Z^{\prime}\right)$ to be considered as well. But as $\bar{V}+\bar{W}$ has been carefully chosen to vanish at infinity at least as fast as $R^{-2}$, values of $Z^{\prime}$ do not become large enough for $\exp \left(i \bar{H}_{e} Z^{\prime}\right)$ to cause trouble.

Using Eq. (12) for $\exp \left(-i \vec{H}_{e} Z\right)$ in Eq. (16) and introducing a conventional pseudostate expansion [Eq. (11)] for $\exp \left(i \bar{H}_{e} Z\right)$, we write $t_{\mathrm{CT}}^{\epsilon_{k}}$, the chargetransfer probability amplitude from the $k$ th bound state of the target atom, as

$$
\begin{aligned}
& t_{\mathrm{CT}}^{\epsilon}=\eta^{-1 / 2}\left(\frac{2}{\sqrt{4 \pi}} f^{3 / 2}\right) \sum_{(n l m)} \int \exp \left[i\left(\lambda_{1}-\lambda_{n}\right) Z\right] \frac{\sin (\bar{\kappa})}{\bar{\kappa}}\left\langle\exp (-f|\overrightarrow{\mathrm{R}}-\overrightarrow{\mathrm{r}}|)\left|\exp \left(i \vec{\lambda}_{2} \cdot \overrightarrow{\mathrm{r}}\right)(\bar{U}+f \bar{W})\right| \phi_{n}^{l}(r) Y_{\imath m}(\hat{r})\right\rangle \\
& \cdot\left\langle\phi_{n}^{l}\left(r^{\prime}\right) Y_{l m}\left(\hat{r}^{\prime}\right)\right| \exp \left(i \lambda_{n} Z\right) \Psi_{k}\left(\overrightarrow{\mathrm{r}}^{\prime}, Z\right\rangle d Z,
\end{aligned}
$$

where $\bar{\kappa}=\Delta_{n} a_{N} Z / 2 \hbar v$. We find the matrix element $\left\langle\phi_{n}^{l} Y_{l m} \mid \exp \left(i \lambda_{n} Z\right) \Psi_{k}\right\rangle$ from a time-development $U$ matrix approach. ${ }^{3}$ That is,

$$
\begin{aligned}
\left\langle\phi_{n}^{l} Y_{l m}\right| & \left.\exp \left(i \lambda_{n} Z_{\alpha}\right) \Psi_{k}\left(\overrightarrow{\mathrm{r}}, Z_{\alpha}\right)\right\rangle \\
=\sum_{(n l m)} & \underline{T}_{(n l m)(n l m)}\left(Z_{\alpha}, Z_{1}\right) \\
& \quad \times\left\langle\phi_{n^{\prime}}^{l^{\prime}} Y_{l^{\prime} m^{\prime}} \mid \exp \left(i \lambda_{n^{\prime}} Z_{1}\right) \Psi_{k}\left(\overrightarrow{\mathrm{r}}, Z_{1}\right)\right\rangle .
\end{aligned}
$$

The matrix $\underline{T}$ is defined $\mathrm{as}^{3}$

$$
\underline{T}\left(Z_{\alpha}, Z_{1}\right)=\underline{Q}\left(Z_{\alpha}, Z_{\alpha-1}\right) \underline{Q}\left(Z_{\alpha-1}, Z_{\alpha-2}\right) \cdots \underline{Q}\left(Z_{2}, Z_{1}\right)
$$

where

$$
\underline{Q}\left(Z_{b}, Z_{a}\right)=\exp \left[-i \eta^{-1 / 2} f \int_{z_{a}}^{Z_{b}} \bar{V}^{I} d Z\right],
$$

and the matrix $\bar{V}^{I}$ has elements

$$
\left\langle\phi_{n}^{l} Y_{l m}|(\bar{V}+\bar{W})| \phi_{n^{\prime}}^{l^{\prime}} Y_{l^{\prime} m^{\prime}}\right\rangle \exp \left[i\left(\lambda_{n}-\lambda_{n^{\prime}}\right) Z\right] .
$$

With this procedure we retain the simplicity of a finite coupled-channel calculation and yet have a valid treatment of the continuum resonance contributions. A calculation was also performed in which the $\sin \bar{\kappa} / \bar{\kappa}$ factor was included in the computation of the diagonal matrix elements of the $U$ matrix. No change in either the charge transfer or ionization cross sections occurred, providing explicit numerical verification of the above contention that large $Z$ values, and hence the $\sin \bar{\kappa} / \bar{\kappa}$ factors, are not very important in these integrals.

The sum on $(\mathrm{nlm})$ is taken over all states, with 
$\Delta_{n}$ set to zero for the bound states, which are identified as those having energy below the ionization threshold. The basis functions used to expand the $\phi_{n}^{l}, \phi_{n}^{l}=\sum_{i} d_{n_{0}}^{i} u_{i}^{l}(r)$, were of the form used before, ${ }^{3}$

$$
u_{i}^{l}=r^{l} \exp \left(-\beta \alpha_{i} r\right)
$$

The $\alpha_{i}$ are suitably chosen complex numbers and $\beta$ is an overall scale factor. We always chose our basis so that it has a $\lambda_{n}$ equal to $\lambda_{1}$. That is, there is always a state on resonance in our basis, for each angular momentum $(\mathrm{lm})$. This means that the basis is changed as a function of energy. This is done using the scale parameter $\beta$.

An interesting question is what is the effect on the amplitude $t_{\mathrm{CT}}$ of setting the $\Delta_{n}$ 's to zero. It turns out that it has negligible effect in all intermediate states except the resonant state and the states either side of resonance, which are generally affected much less than the resonant state. The contribution from the resonance state is generally decreased by a factor $\delta$ if the correct averaging over energy is used as opposed to setting the state exactly on resonance and not averaging. From Eq. (17) we estimate $\delta$ as

$$
\delta=f \pi \hbar v / \Delta_{n} a_{N} \cdot
$$

It is instructive to now examine $t_{\mathrm{CT}}$ for its $f$ (or $Z_{p}$ ) dependence by considering $\Psi^{(+)}$to be expanded in a Born series. Heuristically, one can see the following. For charge-transfer processes off resonance, as $\lambda_{1}-\lambda_{k}$ is generally greater than $f$, we obtain a factor $f$ from the $Z$ integral of Eq. (17). In the first Born term this combines with the $f^{3 / 2}$ factor in $t_{\mathrm{CT}}$ that comes from the projectile $K$-shell wave-function normalization to give an $f$ dependence of $f^{5}$ for the cross section for the first Born term from the potential $\bar{U}$. For the potential $W$ there is the $f$ in $f \bar{W}$ that combines with the $f^{3 / 2}$ from the wavefunction normalization to give a similar $f^{5 / 2}$ factor in the amplitude. For $\bar{W}$ the $Z$ integral is of the form (for $R \gg r$ )

$$
\begin{aligned}
& \int_{-\infty}^{+\infty} \frac{\exp \left[-f R+i\left(\lambda_{1}-\lambda_{k}\right) Z\right]}{R} d Z \\
&=2 K_{0}\left\{B\left[\left(\lambda_{1}-\lambda_{k}\right)^{2}+f^{2}\right]^{1 / 2}\right\},
\end{aligned}
$$

which is independent of $f$ off resonance. Thus the first Born amplitude for the potential $W$ also gives an $f^{5}$ dependence in the cross section. The second Born term for this process is on the other hand dominated by the intermediate resonant state (which is always present). Hence the $U$ part of the potential dominates and the factor from the $Z$ integration is now $\pi \hbar v / \Delta_{n} a_{N}$, independent of $f$ as discussed above. The $f$ dependence thus comes from the $f^{3 / 2}$ projectile $K$-shell normalization fac- tor and from an $f$ due to the fact that we are now considering a second-order perturbation [see Eq. (16)]. We thus obtain an $f^{5} f$ dependence in the cross section from the second Born amplitude, the same as from the first Born. It is probably this identical scaling law for the BrinkmanKramers approximation (first Born, $\bar{W}=0$ ) and for the more accurate second-order calculation that allows a suitably renormalized BrinkmanKramers calculation to fit the experimental data so successfully. It should be noted that our conclusions differ from Lapicki and Losonsky ${ }^{15}$ who have used the idea of increased binding to estimate effects beyond the first Born. This approach for small $f$ gives a correction of the order of $f^{6}$ to the Brinkman-Kramers cross section, in exactly the same way as it gives an $f^{3}$ correction to the first Born $\left(f^{2}\right)$ estimate for ionization.

For resonant charge transfer, the situation is different. Here the first Born $U$-potential term dominates, and the resonance factor $f /\left[f^{2}+\left(\lambda_{1}\right.\right.$ $\left.\left.-\lambda_{n}\right)^{2}\right]$ must be handled with care. To calculate charge transfer to the whole resonance region of the continuum of the target we must square this Born amplitude and integrate with respect to continuum state energy. To do this we can use our . new integration scheme, but must average after we square the $Z$ integral, not before. This energy averaging gives a $f^{-1}$ factor, which combines with the $f^{3}$ from the square of the projectile bound state wave-function normalization to give probabilities at each impact parameter that scale as $f^{2}$. For the cross-section $f$ scaling one must also note that the $e^{-f B}$ large $B$ dependence of the resonant amplitude gives an additional $1 / f^{2}$ dependence. Thus with all these effects combined, the resonant cross section should be approximately independent of $f$. This gives an approximate behavior independent of $Z_{p}$ for the dominant contribution to electron stripping from a light projectile by a heavy target.

So far we have concentrated our discussion on those aspects of our calculation which differ from the generally used treatments of charge transfer. Even without the complications produced by continuum resonance effects we still have the problem of evaluating the integrals for $t_{\mathrm{CT}}$ in a rapid, accurate, and tractable manner. To elucidate our approach to this we give some technical, though important, discussion of the calculational procedure in the Appendix. In Sec. IV we discuss our results.

\section{RESULTS}

The number of experiments performed in which charge transfer is directly measured or thought to be of importance is growing at a rapid rate and 
TABLE II. Demonstration of convergence in the number of basis states; $6-\mathrm{MeV} / \mathrm{amu} \alpha$ particles on titanium. Charge capture cross sections for specific target initial states (in units of $10^{-21} \mathrm{~cm}^{2}$ ).

\begin{tabular}{ccccc}
\hline Basis & $1 s$ & $2 s$ & $2 p_{1}$ & $2 p_{0}$ \\
\hline $7 s, 7 p$ & 0.1335 & 1.148 & 3.425 & 4.394 \\
$9 s, 9 p$ & 0.0989 & 1.004 & 3.302 & 4.809 \\
$11 s, 11 p$ & $\mathbf{0 . 0 9 7 0}$ & $\mathbf{1 . 0 4 4}$ & 3.352 & 4.558 \\
\hline
\end{tabular}

it has thus been difficult to choose which type of calculations it is most useful to perform first. In this preliminary work we decided to limit ourselves to answering some simple (we hope global) questions about charge transfer that seem most frequently to arise in discussions: How does charge transfer vary as a function of energy and as a function of projectile charge? How does the cross section compare to the Brinkman-Kramers estimate? What is the projectile charge dependence for electron stripping from a light projectile, etc.?

To answer these questions it has been necessary to perform large numerical calculations, and now we must address the problems of reaching conver gence and of avoiding computational errors. The exchange matrix elements were checked by evaluating the Brinkman-Kramers hydrogenic amplitude analytically and numerically. The HartreeFock potential construction in our basis was checked by using both the post and prior form in the Brinkman-Kramers $\left(t_{U, B}^{E}\right)$ expression. As we have seen, in the small $f$ limit [Eq. (17)] analytical approximations may be made to simplify the expressions involved. In work not yet published, Fitchard ${ }^{16}$ developed these methods to produce an analytic second Born calculation for charge transfer. As a test our code was run for first and second Born terms only and compared to a similar calculation following Fitchard's development. In all these tests satisfactory agreement was found.

Convergence in the number of basis states is of prime importance in work such as this. Here we have included only $s$ and $p$ states in the calculation of $\Psi^{(+)}$. We were guided in this by our experience with the ionization calculation, ${ }^{3}$ where in similar asymmetric collision circumstances $d$ states made less than $5 \%$ difference in the cross sections. Further, they only needed to be included in the first Born terms, and played very little role as intermediate states. A full examination of the role of $d$, and higher angular-momentum states, in our treatment of charge transfer is in progress and will be presented in a later paper. The restriction for the present to $s$ and $p$ states is thus admittedly one aspect of our calculation where convergence has not yet been demonstrated.

In the $s-p$ space we used basis sets of 7,9 , and 11 states for each $m$ value, e.g., $7 s, 7 p m=0$, and $7 p m=1$ states. An example of convergence is given for 6-MeV/amu $\alpha$ particles incident on titanium in Table II. This is for charge transfer from the target $K$ shell and $2 s, 2 p_{1}$, and $2 p_{0}$ com-

TABLE III. Effect of the treatment of the resonance contribution; 2.5-MeV protons on argon. Charge capture cross sections for specific target initial states (in units of $\left.10^{-21} \mathrm{~cm}^{2}\right)$.

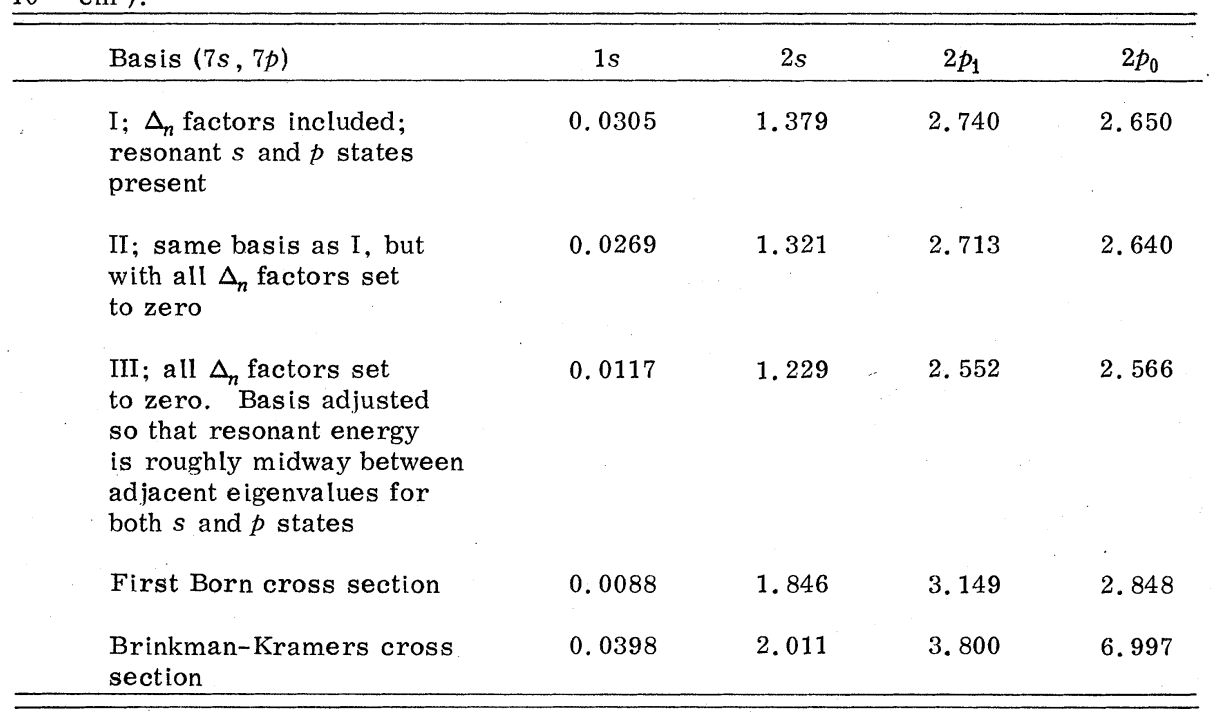


TABLE IV. Energy dependence of target $K$ - and $L$-shell charge-transfer cross sections. $\alpha$-particles on titanium; $(9 s, 9 p)$ basis; cross sections in units of $10^{-22} \mathrm{~cm}^{2}$.

\begin{tabular}{|c|c|c|c|c|c|c|}
\hline \multirow[b]{2}{*}{$E(\mathrm{MeV} / \mathrm{amu})$} & \multicolumn{3}{|c|}{$K$-shell } & \multicolumn{3}{|c|}{$L$-shell } \\
\hline & $\sigma_{\mathrm{CT}}$ & $\begin{array}{l}\text { Ratio to } \\
\text { BK }\end{array}$ & $\begin{array}{l}\text { First Born, } \\
\text { for }(U+f W)\end{array}$ & $\sigma_{\mathrm{CT}}$ & $\begin{array}{l}\text { Ratio to } \\
\text { BK }\end{array}$ & $\begin{array}{l}\text { First Born, } \\
\text { for }(U+f W)\end{array}$ \\
\hline 2 & 0.950 & 0.833 & 0.089 & 1935.0 & 0.359 & 2804.0 \\
\hline 4 & 0.994 & 0.348 & 0.619 & 366.2 & 0.474 & 431.6 \\
\hline 6 & 0.989 & 0.305 & 1.151 & 91.2 & 0.524 & 102.9 \\
\hline 8 & 0.929 & 0.322 & 1.279 & 29.0 & 0.544 & 32.7 \\
\hline
\end{tabular}

ponents of the target $L$ shell, to the $K$ shell of the projectile. The convergence is quite comparable with that we found in ionization calculations, and occurs at about the same number of basis states.

An important new feature of our method is the expansion of $\exp \left(i H_{e} t / \hbar\right)$ given in Eq. (12). In this way we feel we properly, or at least more properly, include resonance effects in our pseudostate approach. The extent that this special treatment of the resonance is important is illustrated in Table III, which gives charge transfer cross sections for 2.5-MeV protons on argon. In Table III, results are given for our calculation, including the $\Delta_{n}$ factors as previously described, and for an identical calculation except that all $\Delta_{n}$ 's [or the $\kappa$, see Eq. (18)] were set to zero. The comparison between these two results shows the effect of not averaging the resonance contribution, i.e., the result a conventional calculation (if set on resonance) would produce. The third set of results in Table III are for a calculation using a similar basis, but with the scale factor in the basis functions adjusted to place the resonance energy approximately midway between adjacent eigenvalues for both the $s$ and $p$ pseudostates. This basis thus causes the resonance effect to be essentially missed altogether. Comparison between calcula- tions II and III shows the fluctuations one could expect as the basis set is varied if no attention is paid to the resonance. For the $K$ shell this is seen to amount to over a factor of 2 . Without the proper treatment of the resonance effect the convergence exhibited in Table II could not have been obtained. Also note that for these processes, the resonance only appears in second-and higher-order Born terms. The first Born results are given in the next to last row of Table III, and one can see that the effect of the resonance treatment on the corrections to the first Born are large indeed. [Here, as for all results presented in this section, $\bar{W}(R)$ $\left.=\left(1-e^{-R}\right) / R_{0}\right] \mathrm{A}$ similar study was also made for $6-\mathrm{MeV} / \mathrm{amu} \alpha$ particles on titanium, in a $7 s, 7 p$ basis. There setting the $\Delta_{n}$ 's to zero caused the target $K$-shell cross section to change from 0.134 $\times 10^{-21} \mathrm{~cm}^{2}$ to $0.159 \times 10^{-21} \mathrm{~cm}^{2}$; the first Born term is $0.118 \times 10^{-21} \mathrm{~cm}^{2}$. Note that in this case setting the $\Delta_{n}$ to zero caused an increase in the cross section, and in the former a decrease. This illustrates that the $s$ and $p$ resonance terms interfere with each other, and with other higher-order Born terms. We also point out that by the earlier discussions, the resonance treatment may well be expected to be even more important for small $f$, that is for greater nuclear charge asymmetry be-

TABLE V. $Z_{p}$ dependence of target $K$ - and $L$-shell charge-transfer cross sections; fully stripped 6.07- MeV/amu He, C, and O on copper; $(9 s, 9 p)$ basis.

\begin{tabular}{cccccc}
\hline \multicolumn{7}{c}{$K$-shell; cross sections in units of $10^{-24} \mathrm{~cm}^{2}$} \\
$Z_{p}$ & $\sigma_{\mathrm{CT}}$ & $\sigma_{\mathrm{C} \mathrm{T}} / Z_{p}^{5}$ & $\sigma_{\mathrm{Born}} / Z_{p}^{5}$ & $\sigma_{\mathrm{B} \mathrm{K}} / Z_{p}^{5}$ & $\sigma_{\mathrm{C} \mathrm{T}} / \sigma_{\mathrm{B} \mathrm{K}}$ \\
\hline 2 & 12.85 & 0.402 & 0.170 & 0.991 & 0.406 \\
6 & 2792 & 0.359 & 0.173 & 1.144 & 0.314 \\
8 & 12140 & 0.370 & 0.194 & 1.290 & 0.287 \\
& \multicolumn{5}{c}{$L-$ shell; cross sections in units of $10^{-22} \mathrm{~cm}^{2}$} \\
2 & 83.31 & 2.603 & 3.232 & 5.336 & 0.488 \\
6 & 8972 & 1.154 & 1.309 & 3.523 & 0.328 \\
8 & 21440 & 0.654 & 0.681 & 2.336 & 0.280 \\
\hline
\end{tabular}


TABLE VI. Comparison of calculated $K$-vacancy production cross sections to experiment. Fully stripped $6.07-\mathrm{MeV} / \mathrm{amu} \mathrm{He}, \mathrm{C}$, and $\mathrm{O}$ on copper.

\begin{tabular}{ccccc}
\hline \hline & & \multicolumn{2}{c}{$R=\sigma_{K V}\left(Z_{p}\right) / Z_{p}^{2} \sigma_{K V}(1)$} \\
$Z_{p}$ & $\sigma_{I}$ (in barns) & $\sigma_{I} / Z_{p}^{2}$ (in barns) & Calculated & Experimental ${ }^{\text {a }}$ \\
\hline 2 & 1753 & 438 & 0.986 & $1.03 \pm 0.01$ \\
6 & 14290 & 397 & 1.081 & $1.17 \pm 0.02$ \\
8 & 24400 & 381 & 1.326 & $1.33 \pm 0.02$ \\
\hline
\end{tabular}

${ }^{a}$ Reference 17.

tween the collision partners.

We will now make a brief comparison between these $p$-Ar target $K$-shell to projectile $K$-shell and target $L$-shell to projectile $K$-shell cross sections and the experiments of Macdonald et $a l,{ }^{8}$ who observed the cross section for charge transfer to the projectile accompanied by production of a $K$ shell hole in the target. However, if the requirement that the system wave function be antisymmetric in the electron coordinates (space and spin) is imposed, then the expression in the independent particle model for the cross section of simultaneously producing the charge-transferred state and the $K$-shell hole involves terms in addition to that corresponding to target $K$-shell to projectile $K$ shell charge transfer. This is discussed in detail in a recent paper by two of the present authors, ${ }^{7}$ and was already mentioned in Sec. III. Proper calculations are planned, as well as an investigation of the full energy region covered by the experiment. These calculations will also be compared to the more recent experiments of Cocke et al. ${ }^{8}$ who made similar measurements for other (smaller $Z_{N}$ ) targets. Nonetheless, our $K$-shell to $K$-shell cross section for $2.5-\mathrm{MeV}$ protons is 30.5 barns, whereas the experimental number is 19.2 \pm 3.3 barns. Our total capture cross section, summed over the $K$ and $L$ shells is 6800 barns. The experimental value is 4500 barns. ${ }^{8}$ For the total capture cross section, as opposed to charge transfer accompanied by hole production, our procedure should be correct (within the independentparticle model). We note that our ratio of capture from the target $K$ shell to total capture is 0.0045 , which is in good agreement with the experimental value of $0.005 \pm 0.001 .^{8}$

TABLE VII. Resonance (electron stripping) cross sections and impact-parameterdependent probabilities. $6.07-\mathrm{MeV} / \mathrm{amu} \mathrm{He}, \mathrm{C}$, and $\mathrm{O}$ on copper; $(9 s, 9 p)$ basis.

Probabilities

\begin{tabular}{ccccc}
$B$ & $Z_{p}$ & full $\left|t_{\mathrm{CT}}\right|^{2}$ & BK first Born $\left|t_{U, B}\right|^{2}$ & Full first Born $\left|t_{U, B}+t_{W, B}\right|^{2}$ \\
\hline $0.2468 a_{0} / Z_{N}$ & 2 & 0.0946 & 0.0939 & 0.1024 \\
& 6 & 0.6029 & 0.8661 & 0.7862 \\
$3.857 a_{0} / Z_{N}$ & 8 & 0.5946 & 1.3703 & 0.9208 \\
& 2 & 0.0788 & 0.0794 & 0.0820 \\
& 6 & 0.4067 & 0.5032 & 0.4502 \\
$11.30 a_{0} / Z_{N}$ & 8 & 0.4178 & 0.6827 & 0.4724 \\
& 6 & 0.0356 & 0.0354 & 0.0358 \\
& 8 & 0.0313 & 0.0320 & 0.0319 \\
& & 0.0152 & 0.0180 & 0.0156
\end{tabular}

Cross sections; in units of $10^{-20} \mathrm{~cm}^{2}$.

\begin{tabular}{ccc}
$Z_{p}$ & $\sigma_{\mathrm{CT}}^{E}$ & BK first Born, $\sigma_{U, B}$ \\
2 & 191.9 & 191.9 \\
6 & 256.5 & 302.2 \\
8 & 219.6 & 348.0 \\
\hline
\end{tabular}


Table IV illustrates the energy dependence of our cross sections for $\alpha$ particles incident on titanium, and Table $\mathrm{V}$ the $Z_{p}$ dependence for various projectiles at $6.07 \mathrm{MeV} / \mathrm{amu}$ incident on copper. The cross sections are generally a factor of $0.3-$ 0.5 times less than the Brinkman-Kramers (BK) estimate. This means that for $\alpha$ particles on titanium at the higher energies, our target $K$-shell charge-transfer cross sections are smaller than previously postulated ${ }^{3}$ on the basis of ionization calculations and experiments on the projectile charge dependence of target $K$-shell hole production. This previous estimate was that charge transfer should be at least as large as BrinkmanKramers. In Table IV the sudden increase of the ratio to $\mathrm{BK}$ at $2 \mathrm{MeV} / \mathrm{amu}$ is a reflection of the fact that at this energy the $\mathrm{BK}$ cross section has started to rapidly decrease as the energy is decreased, whereas ours has not. That is, the peaks in the cross sections are somewhat offset. Table $\mathrm{V}$ shows that our cross sections, like the Brinkman-Kramers, scale approximately as $Z_{p}^{5}$. These tables also show the extent to which our first Born term is a good approximation to the actual cross section. For $\alpha$ particles on titanium the agreement is good except for the lowest energy. For the various projectiles on copper at $6.07 \mathrm{MeV} /$ amu the full calculation and the first Born agree to within a factor of 2 for the $K$ shell, and much closer for the $L$ shell.

In Table VI the charge transfer results of Table $\mathrm{V}$ are combined with ionization cross sections $\sigma_{\mathrm{I}}$, obtained in the same calculation, to obtain $K$-shell vacancy production cross sections, $\sigma_{K V}=\sigma_{I}+(1$ $\left.+\frac{1}{8}\right) \sigma_{\mathrm{CT}}$. The factor $\left(1+\frac{1}{8}\right)$ is included to account approximately for charge transfer into the excited bound states of the projectile. For this projectile velocity and target the $\rho$ of Ref. 2 is negative ( $\rho$ $\approx-0.02$ ), so without the inclusion of the chargetransfer contribution to the vacancy production the ratio $R=\sigma_{K V}\left(Z_{p}\right) / Z_{p}^{2} \sigma_{K V}(1)$ would be very much less than one for the $\mathrm{C}$ and $\mathrm{O}$ projectiles. With our charge transfer included, the agreement with the experiment of Watson et al. ${ }^{17}$ is fair.

In Table VII we present some sample results for the cross section, and impact-parameter-dependent probability, for transitions between the $K$ shell of the projectile and the continuum pseudostates $(s$ and $p)$ that are at the resonance energy. The results we have chosen to present are for fully stripped $\mathrm{He}, \mathrm{C}$, and $\mathrm{O}$ projectiles at $6.07 \mathrm{MeV} /$ amu incident on copper. As pointed out in Sec. III, for these resonance processes we must average any sharp resonance features in the probability. These sharp features in the probability are present only in the first Born $U$-potential contribution $\left|t_{U, B}^{E}\right|^{2}$, and this term was energy averaged in the same scheme developed in Sec. II and used in the amplitudes. Note that the first Born cross section $\sigma_{U, B}$, for which the $Z$ integrations may be done analytically, is a very good approximation to the true cross section at small $Z_{p}$, and is still reasonable at $Z_{p}=8$. The same thing is true of the impact-parameter-dependent probabilities. These BK first Born probabilities show the $Z_{p}^{2}$ scaling discussed in Sec. III. The cross sections themselves do turn out to be rather insensitive to the value of $Z_{p}$, as the scaling arguments predicted. Table VII also shows the more rapid falloff of the probability with $B$ that one has as $f$ is increased. Note also that at small $B$ and the larger $Z_{p}$, the probability of electron removal from the projectile to the continuum of the target is close to unity. Finally, we note that the target resonance state dominates the total electron removal cross section by several orders of magnitude for $Z_{p}=2$, but is only about half the total for $Z_{p}=8$.

\section{SUMMARY AND CONCLUSIONS}

We have extended our previous efficient method of calculating inner-shell ionization cross sections in asymmetric heavy-particle collisions to include charge transfer. Our method expands the time-dependent wave function in terms of basis functions centered on the target nucleus. As in our ionization calculations, we are able to pretabulate the requisite matrix elements, and then use them for all impact parameters. We have developed an efficient method for evaluating the exchange matrix elements; the method is appropriate for the asymmetric collisions we are considering. Our calculation simultaneously produces excitation, ionization, and charge-transfer amplitudes, so it is well suited to future extension to treatment of multiple ionization and ionization accompanied by charge transfer.

An essential new feature of the present work is the recognition of the importance of target continuum states the energy of which is equal to the kinetic energy, in the target frame, of the electron on the projectile, and the development (Sec. II) of a method of treating such resonance effects in our pseudostate calculation. Only with this resonance properly treated could the basis set convergence achieved in this calculation have been obtained.

In this initial paper on charge transfer we have presented selected numerical results that demonstrate our method, and that show the general trends of our results, for $Z_{N} / Z_{p}$ in the range 4-18. In particular, we show that for $\alpha$ particles on titanium in the energy range 2-8 MeV/amu, for 6.07-MeV/amu fully stripped $\mathrm{He}, \mathrm{C}$, and $\mathrm{O}$ on 
copper, and for $2.5-\mathrm{MeV}$ protons on argon the target $K$ - and $L$-shell charge-transfer cross sections are generally 0.3-0.5 times the Brinkman-

Kramers estimate. These cross sections all scale approximately as $Z_{p}^{5}$. That our supposed accurate cross sections are in a roughly constant ratio to Brinkman-Kramers over this rather wide range of energies and projectile-target combinations illustrates why empirically scaled BK cross sections have been found to give good fits to experiment. For $\alpha$ particles on titanium our $K$-shell charge-transfer cross sections, being 0.3 or so times BK, are somewhat less than we estimated earlier ${ }^{3}$ on the basis of hole-production experimental data and our ionization calculations. The small but persistent discrepancy between our calculations and experiment for $K$-shell hole production thus remains a puzzle. For the various projectiles on copper, our calculation is in fair agreement with the $K$-shell hole production measurements of Watson et al.,$^{17}$ and the charge transfer is a very appreciable part of our hole-production cross sections. We have also compared our target $K$-shell to projectile $K$-shell charge-transfer cross sections for $2.5-\mathrm{MeV}$ protons on argon to the experimental results of Macdonald et al.,$^{8}$ but noted that a recent paper on the effects of antisymmetry in the independent-particle model shows that other amplitudes are involved in a complete theoretical description. ${ }^{7}$

We have noted that the time-reversed process of charge transfer from the projectile $K$ shell to the resonance continuum state of the target is a dominant electron stripping mechanism, especially for the smaller $Z_{p} / Z_{N}$. Such cross sections have a much weaker $Z_{p}$ dependence, the probability at a given impact parameter scaling as $Z_{p}^{2}$ in the small $Z_{p} / Z_{N}$ limit and the cross sections being roughly independent of $Z_{p}$. Sample results were presented for fully stripped 6.07-MeV/amu He, $\mathrm{C}$, and $\mathrm{O}$ projectiles on copper. We also noted, and explicitly showed in our calculated results, that the Brinkman-Kramers cross section dominates for this process. This Brinkman-Kramers probability at each impact parameter can be, and is, calculated analytically.

Our results have so far been restricted to only $s$ and $p$ angular-momentum states in the targetcentered expansion of $\Psi^{(+)}$. Removal of this restriction is currently underway, as are also calculations of multiple ionization and ionization accompanied by charge-transfer processes.

\section{ACKNOWLEDGMENT}

Work supported by the Center for Energy and Mineral Resources, Texas A \& M University.

\section{APPENDIX}

The wave function $\Psi^{(+)}$can be calculated very quickly, as in our previous ionization calculations using the $U$-matrix approach. ${ }^{3}$ In a one-centered expansion for the wave function the direct matrix elements of $\bar{V}+\bar{W}$ taken between eigenstates $\phi_{n}^{l}(r) Y_{l m}(r)$ consist of a sum of analytic terms, functions of $R$ only, combined with quickly calculable factors such as $B / R, Z / R$, etc. The individual terms can be stored in the computer as functions of $R$, and the full matrix element reconstructed as needed for each value of $Z$ and $B$. This is not the case with the so-called exchange matrix elements $M_{i, l m}$

$$
\begin{aligned}
M_{i, l m}(B, Z)=\int & \exp \left[-f|\overrightarrow{\mathrm{R}}-\overrightarrow{\mathrm{r}}|-\alpha_{i l} r+i \vec{\lambda}_{2} \cdot \overrightarrow{\mathbf{r}}\right] \\
& \times[\bar{U}+f \bar{W}] Y_{l m}(\hat{r}) r^{l} d^{3} r
\end{aligned}
$$

The integral here cannot be evaluated analytically, and if it could be it still would not be a function of $R$ only, but rather a function of $B$ and $Z$. But even more serious it should be observed that $M_{i, l m}(B, Z)$ has a range in $R$ which is of the order $f^{-1}$, i.e., it falls to zero very slowly as $R \rightarrow \infty$. Subsequent integrations on the variable $Z$ with oscillatory factors such as $\exp \left[i\left(\lambda_{1}-\lambda_{n}\right) Z\right]$ can be very tedious, and unless special care is taken $M_{i, l m}(B, Z)$ might need to be evaluated at a large number of $Z$ points, say 400 , to obtain $t_{\text {CT }}$ accurately. It is just these difficulties that led us away from the use of a two-centered expansion in . the first place, and we must now demonstrate how to solve them.

We used the following treatment of $M_{i, l m}(B, Z)$. Our first step is to exhibit the angular dependence of $\exp (-f|\vec{R}-\overrightarrow{\mathbf{r}}|)$ by formally expanding it in a complete set of spherical harmonics

$\exp (-f|\overrightarrow{\mathrm{R}}-\overrightarrow{\mathrm{r}}|)=\sum_{L, M} Y_{L M}^{*}(\hat{\mathrm{r}}) Y_{L M}(\hat{R}) a_{L_{0} M}(R, r)$.

To cast the $\overrightarrow{\mathbf{r}}$ integration in $M_{i_{0} l m}$ into a convenient form, we evaluate the $\vec{r}$ integration in the following manner. The $\phi_{r}$ integration gives $M=m$. We next evaluate the remaining integral on $x=\cos \theta_{r}$, which is of the form

$$
V M=r^{l} \int_{-1}^{+1} P_{L M}(x) P_{l m}(x) \exp \left(i \lambda_{2} r x\right) d x
$$

by numerical quadrature. ${ }^{18}$ We take care to do so in such manner as to lead to an expression of the form

$$
V M=r^{L} \sum_{j} b_{j}^{L l m} \exp \left(-\beta_{j}^{L l m} r\right) .
$$

The form of this integrand is important as the $r$ integration

$$
\int_{0}^{\infty} a_{L, M}(R, r)(\bar{U}+f \bar{W}) \exp \left[-\left(\alpha_{i l}+\beta_{j}\right) r\right] r^{L+2} d r
$$


can be performed exactly. ${ }^{19}$ This procedure leads to a form for $M_{i_{\imath} l m}(B, Z)$ of

$$
M_{i_{0} \imath m}(B, Z)=\sum_{L} Y_{L M}(\hat{R}) C_{L, \imath m}^{i}(R)
$$

We have thus, for our one-centered basis, cast the exchange matrix elements into the form of functions, of $R$, that can be precomputed and then used for all $B$, multiplied by easily calculable functions of $B$ and $Z$, the $Y_{L m}(\hat{R})$. Detailed tables of the matrix elements are available. ${ }^{19}$ The elements $C_{L, \imath m}^{i}(R)$ are used to construct matrix elements for the pseudostates,

$$
C_{L, \imath m}^{n}(R)=\sum_{i} d_{n, l}^{i} C_{L_{0} \iota m}^{i}(R)
$$

and

$$
M_{l m}^{n}(\overrightarrow{\mathrm{R}})=\sum_{L} C_{L_{\mathrm{v}} \imath m}^{n}(R) Y_{\imath m}(\hat{R})
$$

where

$$
\phi_{n}^{l}(r)=\sum_{i} d_{n, l}^{i} r^{l} \exp \left(-\alpha_{i l} r\right)
$$

The matrix elements $C_{L, l m}^{n}(R)$ are stored at the set of integration points used for $R$, and the $M_{l m}^{n}(\overrightarrow{\mathrm{R}})$ constructed as needed.

The sum on $L$ of Eq. (30) is quite rapidly convergent, and less than a $15 \%$ error in the cross sections is made by using only $L=0$ and 1 . However, in most calculations we used a maximum $L$ of 3 and tested convergence by using $L=4$, which produced a change in the final answers of less than $0.5 \%$. In the sum of Eq. (27) up to 60 points were used to establish satisfactory convergence. The typical number of points used for the present calculations was 28 for $L=0,34$ for $L=1,40$ for $L$ $=2$, and 46 for $L=3$.

The initial setting up of these matrix elements takes roughly half of the total running time of the computer program. But once stored they can be used at any value of $B$ and $Z$, subject, of course, to the condition that $\left(B^{2}+Z^{2}\right)^{1 / 2}=R$, where $R$ is an integration point. Note that since we alter the basis for each projectile energy, to bring one of the continuum pseudostates into resonance and because of the $\lambda_{2}$ dependence of the matrix elements, the $C_{L, l m}^{n}$ must be recomputed for each projectile energy. Note also that these matrix elements must be recomputed when $Z_{p}$, or $f$, is changed.

The above treatment of the exchange matrix elements greatly speeds the computation, but in itself is not enough to eliminate completely the considerable computational time that has been associated with the charge-transfer problem since its inception. The difficulty lies in the number of integration points needed to perform the next step, the $Z$ integration. If we had to construct the $C_{L, m}^{n}(R)$ at, say, 400 points instead of the typical 30 or 40 needed in our treatment of the ionization problem, then our program for charge transfer would be 10 times slower, and therefore only 10 times or so faster than conventional two-centered methods. ${ }^{2}$ The expenditure of such computer time may be justifiable for the fundamental proton-hydrogen system, but it is not practical for the many projectile-target arrangements of interest here.

We achieve another order of magnitude savings by the following treatment of the $Z$ integrations. Using time-reversal symmetry we write all integrals involving $\Psi^{(+)}$[Eq. (18)] in the form of either

$$
\int_{0}^{\infty} E(Z) d Z \int_{0}^{\infty} D\left(Z^{\prime}\right) d Z^{\prime}
$$

or

$$
\int_{0}^{\infty} E(Z) d Z \int_{z}^{\infty} D\left(Z^{\prime}\right) d Z^{\prime} .
$$

The analysis closely parallels that used previously in our ionization calculations. Here by $E(Z)$ we refer to integrals containing exchange matrix elements of $\bar{U}+f \bar{W}$ of the type discussed above. By $D(Z)$ we mean integrals involving only one-centered matrix elements of $\bar{V}+\bar{W}$, such as appear in the $U$-matrix [Eq. (20)]. Explicitly, the expression for $t_{C T}^{\epsilon_{n}}$ obtained, when the initial target state has angular-momentum quantum numbers $l$ and $m$, is

$$
\begin{aligned}
& t_{\mathrm{CT}}^{\epsilon_{n}}=\eta^{-1 / 2}\left(\frac{2}{\sqrt{4 \pi}} f^{3 / 2}\right)(-1)^{l-m} \sum_{(n l m) \cdots}\left\{\sum_{(n l m) \cdots \prime}\left(I_{(n l m) \cdots}+J_{(n l m) \cdots}\right)(-1)^{l^{\prime \prime}-m^{\prime \prime}} T_{(n l m) \cdots(n l m) \cdots}(\infty, 0)\right. \\
& \left.+\left[\sum_{(n l m) \cdots}\left(I_{(n l m) \ldots, \ldots}+J_{(n l m) \cdots}\right) T_{(n l m) \cdots(n l m) \cdots}(\infty, 0)\right]^{*}\right\} T_{(n l m)(n l m) \cdots}(\infty, 0) .
\end{aligned}
$$


Here

$$
\begin{aligned}
J_{(n l m)}(B)= & \int_{0}^{\infty} M_{l m}^{n}(B, Z) \frac{\sin (\bar{\kappa})}{\bar{\kappa}} \\
& \times \exp \left[i\left(\lambda_{1}-\lambda_{n}\right) Z\right] d Z,
\end{aligned}
$$

and

$$
\begin{aligned}
I_{(n l m)}(B)=\sum_{(n l m)} \int_{0}^{\infty} M_{l^{\prime} m^{\prime}}^{n^{\prime}}(B, Z) \frac{\sin (\bar{\kappa})}{\bar{\kappa}} \\
\times \\
\times \exp \left[i\left(\lambda_{1}-\lambda_{n^{\prime}}\right) Z\right] \\
\times\left(T_{(n l m)(n l m),}^{*}(\infty, Z)\right. \\
\\
\left.-\delta_{(n l m) \cdot(n l m)}\right) d Z
\end{aligned}
$$

where $\bar{\kappa}=\Delta_{n} a_{N} Z / 2 \hbar v$, as before.

The exchange integral $J_{(n l m)}$ [the one in Eq. (31a)] is the difficult one containing extended integrands. Note that the real part of $J_{(n l m)}$ for $l-m$ even and the imaginary part for $l-m$ odd that come from the $\bar{U}$ part of $\bar{U}+f \bar{W}$ are the same as arise in the Brinkman-Kramers amplitude. These integrals over $Z$ can be and were evaluated analytically. The resulting expressions are in terms of modified Bessel functions $K_{\nu}(c B)$. The $\bar{W}$ part of these integrals are somewhat less troublesome to do numerically, as they fall off faster in $R$. Thus we have an analytic evaluation of the first Born amplitude $t_{U, B}^{n}$, and, we feel, a very accurate evaluation of $t_{W, B}^{n}$. The parts of the exchange integral $J_{(n l m)}$ not done analytically are integrated numerically with a six-point integration rule. The direct integrals required in computing the $U$ matrix are evaluated by using a second-order polynomial, as was done in our previous ionization work. ${ }^{3}$ The remaining integral $I_{(n l m)}$ [or Eq. (31b)] has been constructed to go to zero for large $Z$, and is therefore untroublesome. It was evaluated by the above rule, using a first-order polynomial. The important feature of this scheme is that $\Psi^{(+)}(\overrightarrow{\mathrm{r}}, Z)$ need only be known at 40 or 50 points in $Z$ to accurately evaluate the integrals.
${ }^{1}$ M. C. Brinkman and H. A. Kramers, Proc. Acad. Sci. Amsterdam 33, 973 (1930); D. R. Bates, Proc. R. Soc. Lond. A 247,294 (1958); I. M. Cheshire, Proc. Phys. Soc. Lond. 84,89 (1964); J. Macek, Phys. Rev. A 1,235 (1970); A. Salop and R. E. Olson, ibid. 16, $18 \overline{11}$ (1978).

${ }^{2}$ D. F. Gallaher and L. Wilets, Phys. Rev. 169, 139 (1968); R. S. Shakeshaft, Phys. Rev. A 14, 1626 (1978); C. D. Lin, S. C. Soong, and L. N. Tunnell, ibid, 17, 1646 (1978).

${ }^{3}$ J. F. Reading, A. L. Ford, and E. Fitchard, Phys. Rev. Lett. 36, 573 (1976); A. L. Ford, E. Fitchard, and J. F. Reading, Phys. Rev. A 16, 133 (1977); E. Fitchard, A. L. Ford, and J. F. Reading, ibid. 16, 1325 (1977); G. L. Swafford, J. F. Reading, A. L. Ford, and E. Fitchard, ibid. 16, 1329 (1977).

${ }^{4}$ W. H. Bassichis, J. F. Reading, and R. R. Scheerbaum, Phys. Rev. C 11, 316 (1975); W. H. Bassichis, M. Strayer, J. F. Reading, and R. Scheerbaum, ibid. 18, 632 (1978).

${ }^{5}$ The reason for not using the method described in Ref. 4 is that our basis does not lead to a tridiagonal form for $P H_{e} P$, a crucial, if technical, point in the method.

${ }^{6} \mathrm{~J}$. F. Reading, Phys. Rev. A 8, 3262 (1973).

${ }^{7}$ J. F. Reading and A. L. Ford (unpublished).

${ }^{8}$ C. L. Cocke, R. K. Gardner, B. Curnutte, T. Bratton, and T. K. Saylor, Phys. Rev. A 16, 2248 (1977); J. R. Macdonald, C. L. Cocke, and W.W. Eidson, Phys. Rev. Lett. 32, 648 (1974).

${ }^{9}$ This form was suggested to us by R. Shakeshaft.

${ }^{1 n}$ A. M. Halpern and J. Law, Phys. Rev. A 12, 1776 (1975).

${ }^{11}$ J. D. Jackson and H. Schiff, Phys. Rev. 89 , 359 (1953).

${ }^{12}$ K. Omidvar, J. E. Golden, J. H. McGuire, and L. L. Weaver, Phys. Rev.A 13, 500 (1976); Y. Band, Phys. Rev. Lett. 37, 634 (1976).

${ }^{13}$ E. Clementi and C. Roetti, At. Data Nucl. Data Tables 14, 177 (1974).

${ }^{14} \mathrm{R}$. M. Drisko, thesis (Carnegie Institute of Technology, 1955) (unpublished).

${ }^{15} \mathrm{G}$. Lapicki and W. Losonsky, Phys. Rev. A $\underline{15}, 896$ (1977).

${ }^{16} \mathrm{~A}$. Fitchard (unpublished).

${ }^{17} \mathrm{C}$. W. Lewis, R. L. Watson, J. B. Natowitz, and T. L. Mardt, in Proceedings of the International Conference on Inner Shell Ionization Phenomena, edited by R. W. Fink, S. T. Manson, J. M. Palms, and P. V. Rao. (U.S, AEC, Oak Ridge, Tenn., 1973), p. 1682.

${ }^{18}$ A. Ralston, A First Course in Numerical Analysis (McGraw-Hill, New York, 1965).

${ }^{19} \mathrm{G}$. L. Swafford, Ph.D. thesis, (Texas A \& M University, 1978) (unpublished). 